\title{
Development And Validation of A Novel Hypoxia- And Immune-Related Prognostic Signature For Bone And Soft Tissue Sarcoma Patients
}

\section{Zhehong Li}

Affiliated Hospital of Chengde Medical College

Junqiang Wei

Affiliated Hospital of Chengde Medical College

Honghong Zheng

Affiliated Hospital of Chengde Medical College

Xintian Gan

Affiliated Hospital of Chengde Medical College

Mingze Song

Affiliated Hospital of Chengde Medical College

Yafang Zhang

Affiliated Hospital of Chengde Medical College

Haiying Cao

Affiliated Hospital of Chengde Medical College

Yu Jin ( $\nabla$ cdyxyjy@126.com )

Affiliated Hospital of Chengde Medical College

\section{Research Article}

Keywords: hypoxia, immune, sarcoma, prognosis, biomarker

Posted Date: July 1st, 2021

DOl: https://doi.org/10.21203/rs.3.rs-639983/v1

License: (c) (1) This work is licensed under a Creative Commons Attribution 4.0 International License.

Read Full License 


\section{Abstract}

Background: Hypoxia- and immune-status play an essential role in tumorigenesis and tumor development. This study sought to build a novel hypoxia- and immune-related signature to evaluate sarcoma patients' prognosis.

Methods: Transcriptome data and clinicopathological characteristics of sarcoma patients were downloaded from the TARGET database. We grouped patients with three clusters by using t-SNE. We defined the three cluster as high-, medium-, and low-hypoxia clusters by K-M analysis and differential expression of target genes associated with the HIF-1 signaling pathway. Then we used the "limma" package to screen hypoxia-related differentially expressed genes (HRDEGs) in the high- and low-hypoxia clusters. We immediately assessed the immune status by using the single sample Gene Set Enrichment Analysis (sSGSEA) and divided the patients into high-, medium-, and low-immune clusters. Immunerelated DEGs (IRDEGs) were filtered in the high- and low-immune groups. The intersection of HRDEGs and IRDEGs screened overlapping genes. We used a combination of Cox regression analysis and LASSO model to obtain prognosis-related genes and established a novel hypoxia- and immune-related prognostic signature for sarcoma patients. Combining clinicopathological characteristics of sarcoma patients, we evaluated the signature by univariate and multivariate Cox regression analysis. We further divided the patients into high- and low-risk groups based on the novel signature. Finally, we evaluated the differences in hypoxia status and the immune status in high- and low-risk groups.

Results: We identified two genes associated with prognosis, CMA1 and IGDCC3. The novel Prognostic signature could be used as an independent prognostic factor for sarcoma patients. We distinguished patients more effectively by their different survival outcomes, immune cells' infiltration status, and immune-related markers.

Conclusion: The hypoxia- and immune-related prognostic signature can be used to stratify the risk of sarcoma patients. Our study established a new prognostic signature and provides a potential prognostic markers for hypoxia- and immune-related therapy.

\section{Introduction}

Sarcomas are highly heterogeneous malignant tumours of mesenchymal origin, with over 70 histological subtypes ${ }^{[1]}{ }^{[2]}$. Sarcomas account for $1 \%$ of all malignancies in adults and approximately $15 \%-21 \%$ in children ${ }^{[2]}[3]$. The five-year survival rate is $60-80 \%$ in sarcoma patients, but for patients with distant

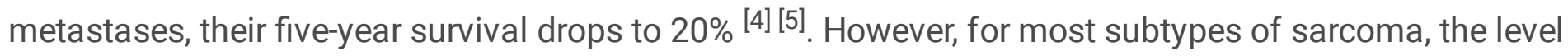
of knowledge about the molecular biology of their occurrence and progression remains limited ${ }^{[6]}$. Insufficient awareness of the molecular mechanisms of sarcoma development often leads to a lack of effective treatments, especially advanced sarcomas. Therefore, it is urgent to further analyze the novel sarcoma' molecular features and to provide new ideas for diagnosis and therapies. 
Hypoxia microenvironment often occurs in tumor, hypoxic foci are formed when the metabolism of tumor cells is greater than the supply of oxygen ${ }^{[7][8]}{ }^{[8]}$. It has been reported that hypoxia can increase the metastatic potential in sarcoma patients ${ }^{[9]}$. The 18 -month progression-free survival (PFS) rate was $70 \%$ for patients with median tumour oxygen pressure $>10 \mathrm{~mm} \mathrm{Hg}$, conversely, the PFS discounting $30 \%$ for patients with median tumour oxygen pressure $<10 \mathrm{~mm} \mathrm{Hg}^{[10]}$. Inadequate oxygen levels in the tumor induce a series of downstream responses that lead to poor patient prognosis. On the other hand, the immune system acts an extremely key role in the human anti-tumor mechanism ${ }^{[11]}$. Immune checkpoint inhibitor (ICl) therapy, including monoclonal antibodies against programmed cell death protein-1/-ligand 1 (PD-1/PD-L1), and cytotoxic T lymphocyte-associated antigen-4 (CTLA-4) has made some significant progress in sarcoma patients ${ }^{[12][13]}$. However, abudant local immune cell infiltration can effectively predicts sarcoma patients better sensitivity to ICI therapy ${ }^{[14]}{ }^{[15]}$. Recently, more and more researches have shown a direct or indirect link between hypoxia and immune status in the sarcoma [16] [17] [18]. However, the specific underlying regulatory mechanism between the hypoxia and immune is unclear.

Therefore, we adopted the single sample Gene Set Enrichment Analysis (SSGSEA) and t-distributed Stochastic Neighbor Embedding (t-SNE) to assess immune and hypoxia status, respectively. We then screened out the intersection fraction of differential genes and constructed a new prognostic model by systematic analysis combining clinicopathological features, immune cells' infiltration status, and immune-related markers, with the an aim of improving sarcoma patients' prognosis.

\section{Methods}

\section{Gene expression datasets collection}

Gene expression datasets and corresponding clinical data for sarcoma patients were downloaded from the Therapeutically Applicable Research To Generate Effective Treatments (TARGET) database (https://ocg.cancer.gov/programs/target). The TARGET database is focused on pediatric tumors and is designed to guide the development of effective, less toxic therapies by identifying data on molecular changes in pediatric tumors. Sarcoma patients with complete follow-up data were included in our study [19]. Clinicopathological characteristics included included age, gender, race, status of surgical margin and distant metastatic status in the study .

\section{Hypoxia-related differentially expressed genes (HRDEGs) and hypoxia status}

We used t-SNE to differentiate patients' hypoxia-status ${ }^{[20]}$. t-SNE is a nonlinear dimensional reduction algorithm, which downscales multidimensional data into two or three dimensions ${ }^{[21]}$. We downloaded hypoxia-related genes (HRGs) set (hallmark gene sets, gene symbols) from the Gene Set Enrichment Analysis (GSEA) database (http://www.gsea-msigdb.org/gsea/index.jsp). Based on HRGs, we grouped the sarcoma patients by t-SNE analysis. Based on the results of t-SNE, we obtained different clusters and the corresponding marker genes. Then we plotted the K-M curves for different clusters. Based on the clustering and K-M analysis results, two clusters (the high- and the low-hypoxia groups) were selected to 
assess hypoxia status. To further evaluate these clusters' hypoxia status, we grouped marker genes into the high- and the low-hypoxia groups through gene ontology (GO) signaling pathway analysis..

Meanwhile, we analyzed changes in the expression of two clusters of hypoxia inducible factor 1 (HIF-1) signaling pathway-related target genes ( 27 genes in total, of which 15 genes increase oxygen delivery and 12 genes reduce oxygen consumption). These target genes were obtained from the Kyoto encyclopedia of genes and genomes (KEGG) database (https://www. kegg. jp/; ID: 04066). Then we used the "limma" package to select HRDEGs. The acquisition criteria for HRDEGs are log fold change (logFC) $>2$ and $p$ value $<0.001$. GO and KEGG pathway analyses were performed for these HRDEGs.

\section{Immune-related differentially expressed genes (IRDEGs) and immune status}

We obtained 29 immune-related cells and types ${ }^{[22]}$. We applied ssGSEA to analyze the transcriptome database of sarcoma patients, to calculate sample ssGSEA scores, to quantify the level of enrichment of

immune cells and immune pathways ${ }^{[23]}$. Then we classified patients into three immune clusters (high-, medium-, and low-immune clusters) based on sSGSEA scores. Estimation of Stromal and Immune cells in Malignant Tumor tissues using Expression (ESTIMATE) were used to validate the ssGSEA subgroup [24]. Calculation and analysis of ESTIMATE score, tumor-purity, immune-score and stromal score for three immune clusters were performed by using the "ESTIMATE" package. Meanwhile, we used the "limma" package to select IRDEGs between high- and low-immune clusters. The acquisition criteria for IRDEGs are $\log F C>1$ and p-value $<0.05$. GO and KEGG pathway analyses were performed for these IRDEGs.

\section{Construction and validation of a novel hypoxia- and immune-related prognostic signature}

The intersection genes of HRDEGs and IRDEGs were taken for the next step analysis. Prognosis-related genes were derived by univariate-multivariate Cox regression analysis and LASSO regression analysis in combination with survival data of sarcoma patients, and a novel hypoxia- and immune-related prognostic model was developed. Patients were divided into high-and low-risk groups according to the best cut-off point. The risk scoring formula is as follows:

\section{Risk score $=\sum_{i}^{n}$ coef $\left(\right.$ prognostic gene $\left.e_{\mathrm{i}}\right) \times \operatorname{expr}\left(\right.$ prognostic gene $\left.e_{\mathrm{i}}\right)$}

coef (prognostic gene $e_{\mathrm{i}}$ ) and expr (prognostic gene $\mathrm{i}_{\mathrm{i}}$ ) respectively represent the survival correlation coefficient and prognostic gene-expression of patients.

We combined the clinicopathological characteristics of the phases and assessed the performance of the signature by Cox regression analysis. perating characteristic (ROC) curves at one-, three-, and five-years were generated by "survival ROC" package. Sarcoma patients in the high- and low-risk groups were analyzed by K-M analysis.

\section{The relationship between prognostic signature and hypoxia and immunity}


The active status of signaling pathways in the high-risk and low-risk groups were determined by GSEA software (GSEA 4.1.0, https://www. gsea-msigdb. org/gsea/index. jsp). GSEA was performed by the gene sets "c2.cp.kegg.v7.2.symbols.gmt". Then, normalized enrichment score (NES) was calculated, and the acquisition criteria for significant were nominal p-value (NOM p-val) $<0.05$ and false discovery rate qvalue (FDR q-val) $<0.25$. The abundance of the six immune cell immune infiltrates in various cancer types were systematically analyzed by TIMER (Tumor Immune Estimation Resource, https://cistrome.shinyapps.io/timer/) ${ }^{[25]}$. Then, we evaluated the relationship between the prognostic signature and the six immune cell infiltration by using the TIMER database. Cibersort is a tool for deconvolution of the expression matrix of immune cell subtypes based on the principle of linear support vector regression ${ }^{[26]}$. We used the Cibersort database to analyze the relationship between 22 immune cells and the prognostic signature. At the same time, we further analyzed the relationship between IClrelated biomarkers (CTLA-4, PD-1/PD-L1, and T-cell immunoglobulin mucin 3[TIM3]) and the prognostic signature.

\section{Statistical analyses}

Statistical analysis was performed using R (i386 4.0.3, Download at https://www.r-project.org/). Unpaired Student's t-test, the Wilcoxon rank-sum test, ANOVA, and the Kruskal-Wallis test were used for the comparison of continuous variables. Qualitative variables were explored using Fisher's exact test or Pearson chi-square test. Differences of $p$-value $<0.05$ indicate statistical significance unless otherwise stated.

\section{Results}

\section{Flowchart and patients}

A detailed workflow is shown in Figure 1. A total of 259 sarcoma patients with complete follow-up data were included in our study, The clinicopathological characteristics of the patients are shown in Table 1. 
Table 1 Baseline characteristics of 259 sarcoma patients

Total set $(n=259)$

\begin{tabular}{lll}
\hline Age, years & & $60.50 \pm 14.33$ \\
\hline Race & White & 227 \\
\hline & Other & 32 \\
\hline Sex & & \\
\hline & Male & 118 \\
\hline Tumor site & Female & 141 \\
\hline & Soft tissue & 115 \\
\hline Other & 144 \\
\hline Margin status & & \\
\hline & Ro & 72 \\
\hline & R1/2 & 137 \\
\hline Unknown & 50 \\
\hline Metastasis & & \\
\hline & No & 118 \\
\hline Yes & 57 \\
\hline & Unknown & 84 \\
\hline
\end{tabular}

\section{HRDEGs and hypoxia status in sarcomas}

Two hundred HRGs are shown in Supplementary Table 1. Altogether 99, 83, 77 sarcoma patients were separately collected into 3 clusters (cluster1-3) by the nonlinear dimensionality reduction algorithm t-SNE (see Figure 2A). Meanwhile, the survival curves of the three clusters are shown in Figure 2B. Previous studies have demonstrated that patients with high-hypoxia in the tumor microenvironment have a worse prognosis than those with low-hypoxia [27] [28]. Therefore, combining the survival curves of the 3 clusters, we hypothesized that patients in cluster 2 were the highest-hypoxia-state and patients in cluster 3 were the lowest-hypoxia-state. The marker genes for the 3 clusters are shown in Supplementary Table 2, and the heat map of the top 10 marker genes for each cluster is shown in Figure $2 \mathrm{C}$. In cluster 2, the marker genes were mainly enriched in molecular function (MF, see Figure 2D, Supplementary Table 3 ) and not in biological processes (BP). While in cluster 3 , the marker genes were mainly enriched in sugar-metabolism- 
related-BP and MF (see Figure 2E, Supplementary Table 4). Among the 15 genes involved in "increased oxygen delivery", 8 (53.33\%) genes were differentially expressed in the highest-hypoxia-state and lowesthypoxia-state group (Supplementary Figure 1A-0). In addition, 10 of the $12(83.33 \%)$ genes associated with "reduced oxygen consumption" were differentially expressed (Supplementary Figure 2A-L). Based on the survival analysis results and correlation analysis of HIF-1 signaling pathway-related proteins, patients in clusters 2 and 3 were defined as "hypoxia-high" or "hypoxia-low" groups. Finally, we obtained 1497 HRDEGs by the "limma" package. Of these, 821 was up-regulated, while 676 was down-regulated, and HRDEGs heat map and volcano map are shown in Fiqure 2F, 2G. GO and KEGG analysis of the HRDEGs are shown in Supplementary Figure 3A, 3B and Supplementary Table 5, 6. "Muscle system process", "Regulation of membrane potential" and "Imuscle contraction" were the most significantly enriched GO items of BP. The top $3 \mathrm{GO}$ terms of cellular component (CC) were "Contractile fiber", "Transmembrane transporter complex" and "Transporter complex". The top 3 GO terms of MF were "Channel activity", "Passive transmembrane transporter activity" and "Ion channel activity". The top 3 KEGG pathways were enriched in "Neuroactive ligand-receptor interaction", "Calcium signaling pathway" and "CAMP signaling pathway".

\section{IRDEGs and immune status in sarcomas}

By ssGSEA analysis and, we categorized 112, 16, 131 sarcoma patients into immune-high, -medium, and low groups (see Figure 3A, 3B). We used the ESTIMATE method to calculate the stromal score, immune score, and tumor purity and mapped the tumor microenvironment heat map (see Figure $3 \mathrm{C}$ ). The results showed that the stromal score and immune score were higher in the immune-high group than in the immune-low group ( $<<0.001$, see Figure 3D, 3E), and the tumor purity score was lower in the immunehigh group than in the immune-low group $(p<0.001$, see Figure $3 F)$. Finally, we obtained 1875 IRDEGs by the "limma" package, of which 950 were up-regulated and 925 were down-regulated, and IRDEGs heat map and volcano map are shown in Figure 3 G, 3H. GO and KEGG analysis of the IRDEGs are shown in Supplementary Figure 4A, 4B and Supplementary Table 7, 8. "Immune response-activating cell surface receptor signaling pathway", "Immune response-activating signal transduction" and "Lymphocyte mediated immunity" were the most significantly enriched GO items of BP. The top $3 \mathrm{GO}$ terms of CC were "External side of plasma membrane", "Plasma membrane signaling receptor complex" and "Immunoglobulin complex". The top 3 GO terms of MF were "Antigen binding", "Immunoglobulin receptor binding" and "Carbohydrate binding". The top 3 KEGG pathways were enriched in "Cytokine-cytokine receptor interaction", "Neuroactive ligand-receptor interaction" and "Viral protein interaction with cytokine and cytokine receptor".

\section{Development of risk scoring signatures and evaluation of predictive models}

131 overlapping genes were obtained for subsequent analysis by taking intersections of HRDEGs and IRDEGs (see Figure 4A and Supplementary Table 9). Two genes named Chymase 1 (CMA1) and IGDCC3 were derived by univariate and multivariate Cox regression analysis and LASSO analysis (see Figure 4B$4 \mathrm{E})$. Based on the CMA1 and IGDCC3, we model novel hypoxia- and immune-related prognostic signature 
for sarcoma patients. The prognostic signature's ROC curves are shown in Figure 4F, with an optimal cutoff point of 1.287. Sarcoma patients were classified into high- and low-risk groups based on the optimal cut-off point. 1-, 3-, and 5-years ROC curves of prognostic signature were shown in Figure 4G, and 1-, 3-, and 5 -years AUC values were $0.701,0.663$, and 0.686 , respectively. According to the results of the K-M analysis, sarcoma patients in the high-risk group had a worse prognosis than those in the low-risk group ( $p$-value $=0.001$, see Figure $4 \mathrm{H}$ )

\section{Relationship between prognostic signature and clinicopathological features}

Clinicopathological characteristics (including age, gender, race, primary site, margin status, and metastasis status) and prognostic signature were analyzed by univariate and multivariate Cox regression analysis (see Figure5A, 5B). The results of multivariate cox regression analysis showed that prognostic signature was an independent prognostic factor (hazard ratio HR: $1.237,95 \%$ confidence intervals Cls: 1.148-1.332, $P<0.001$ ). The ROC curve showed higher AUC values for prognostic signature than for other single clinicopathological characteristics (see Figure $5 \mathrm{C}$ ). The distributions of gene expression, risk score, and survival status were plotted in the novel signature (see Figure 5D).

\section{Different immune-and hypoxia-related statuses in the low-risk and high-risk groups}

Hypoxia and immune-related status were assessed using GSEA. The screening was performed based on the criteria of NOM p-val $₫ 0.05$ and FDR q-val >0.25. Results showed enrichment of four signaling pathways based on the high-risk group, respectively (They are "HEDGEHOG SIGNALING PATHWAY"; "AMINOACYL TRNA BIOSYNTHESIS"; "BASAL CELL CARCINOMA" and "STEROID BIOSYNTHESIS", Supplementary Table 10, Figure 6A, Supplementary Figure 5). 33 signaling pathways were enriched in the low-risk group (Supplementary Table 11, Figure 6B-E, Supplementary Figure 6 and 7).

\section{Differences in immune status between high- and low-risk sarcoma patients}

We evaluated the relationship between the prognostic signature and the six immune cell infiltration using the TIMER database. It indicate that DC (Cor $=-0.139$, $\mathrm{p}$-value $=0.027$ ) was significantly negatively correlated with the risk score (see Figure $7 \mathrm{~A}$ ). The other five types of immune cells (B cells, $\mathrm{CD} 4^{+} \mathrm{T}$ cells, $\mathrm{CD} 8^{+} \mathrm{T}$ cells, Neutrophils, and Macrophages) had no statistical significance with the risk score (see Figure 7B-F). Besides, we used the CIBERSORT algorithm to assess the different immune cell infiltration in the high-risk group versus the low-risk group. The results in this study show that the infiltration degrees of plasma cells ( $p$-value $₫ 0.01$ ), and $C D 8^{+} T$ cells ( $p$-value $₫ 0.001$ ) were higher in the low-risk group than in the high-risk group (see Figure 8A). We further analyzed the differences in expression of common immune checkpoints between the high- and low-risk groups. The results showed

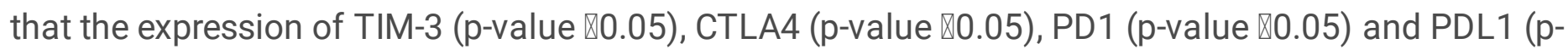
value $₫ 0.001$ ) was higher in the low-risk group than in the high-risk group (see Figure 8B-E).

\section{Discussion}


Hypoxia is a common feature of the tumor microenvironment (TME) which is caused by the high metabolism of tumor cells ${ }^{[29]}\left[{ }^{30]}\right.$. The high metabolism causes the oxygen supply to be relatively less than the oxygen consumption [31] [32]. Hypoxia status is associated with sarcoma metastasis, angiogenesis, radio- and chemo-resistance. It is worth noting that hypoxia can interfere with the immune cells by regulating the expression of immune-related components and co-stimulatory receptors in TME [33]. Research also shown that the HIF-1 signaling pathway correlated with high abundance of macrophages and neutrophils, and decreased T cell levels in Ewing's Sarcoma Family of Tumors ${ }^{[34]}$. At the same time, the changes in the immune microenvironment further affect the hypoxia state of tumor cells. The immune-microenvironment itself is also an important factor in tumorigenesis and tumor progression ${ }^{[35]}$. Due to the complexity of hypoxia and immune in tumors, few immune- and hypoxiarelated markers have been studied in sarcoma patients.

The t-SNE is a clustering and visualization method, which maps high-dimensional to two- or threedimensional while keeping the distribution probabilities of each other as constant as possible ${ }^{[36]}$. On the basis of 200 hypoxia genes, 3 different hypoxia TME patterns were identified by t-SNE. Differentiation of 3 different hypoxia TME patterns by HIF-1 signaling pathway-related target genes and K-M analysis, we then defined high-, medium-, and low-hypoxia groups and looked for HRDEGs. The ssGSEA is an extension of GSEA and the ability to quantify and score pathway enrichment in individual samples [37] [38] Then we classified patients into three immune-clusters (high-, medium-, and low-immune clusters) based on sSGSEA scores. We estimated the score of stromal cells and immune cells in malignant tumor tissues by ESTIMATE, and thus predict tumour purity ${ }^{[39]}$. Comparison of gene expression in patients in high- and low-immune clusters to obtain IRDEGs. 131 overlapping genes were obtained for subsequent analysis by taking intersections of HRDEGs and IRDEGs. Two genes named CMA1 and IGDCC3 were derived by univariate-multivariate Cox regression analysis and LASSO analysis.

We find that CMA1 is an independent protective prognostic factor,conversely, IGDCC3 is an independent non-protective prognostic factor. CMA1 is a pancreatic rennin secreted by mast cells. Functional studies on CMA1 have made some progress in diseases such as hypertension and gastritis, but it has not been studied in sarcoma ${ }^{[40]}[41]$. Shi S's team found significant differences in CMA1 expression levels between tumor tissues and adjacent normal tissues in ten types of tumors such as gastric, breast, and ovarian cancer ${ }^{[42]}$. However, the TIMER database still contains a limited sample size, and Shi S et al. did not include paraneoplastic tissue from sarcomas in the study and did not further explore the expression of CMA1 in sarcomas. ChongA et al. found that the interaction between enhancer 1944 and IGDCC3 could be used to distinguish the $\mathrm{CpG}$ island methylator phenotype in colorectal cancer ${ }^{[43]}$. However, the underlying mechanisms of IGDCC1 in sarcoma progression and immunology are not known. Therefore, our study was the first to propose two genes, CMA1 and IGDCC3, in sarcoma and to establish a hypoxiaand immune-related prognostic signature, while the signature was successfully validated in the cohort. Besides, we used GSEA scores to quantify the activity or abundance of sarcoma samples in high- and low-risk groups for analysis of hypoxia status and immune status. 
With the development of ICI-related treatments (e.g. anti-CTLA4, anti-PD1, anti-PDL1, anti-TIM-3), the treatment of sarcoma continues to be innovative, but, ICl-related resistance in sarcoma patients have been found [44] [45] [46]. Sarcomas is a heterogeneous cancer group and the response to ICl-related treatment varies greatlys ${ }^{[47]}$. Our results showed that the expression of PD-1, PD-L1, TIM-3, and CTLA-4 were lower in the high-risk group than in the low-risk group. It should be noted that ICl-related treatments is only effective in patients with high expression of ICl-related biomarkers ${ }^{[48]}$. Therefore, combined with the K-M analysis of the high- and low-risk groups, our study hypothesized that the low expression of IClrelated biomarkers in the high-risk group led to the ineffectiveness of ICl-related treatment, which further affected the survival prognosis of patients. Interestingly, we used the TIMER and CIBERSORT databases to analyze immune cell infiltration in the high- and low-risk groups, and combining the findings of both databases, the results showed that the infiltration course of $\mathrm{CD}^{+} \mathrm{T}$ cells, plasma cells, and DCs was lower in the high-risk than in the low-risk group. CD8 $+T$ cells, plasma cells and DCs are important

components of the immune system and are important components in the anti-tumor mechanism [47] [49]. Based on the importance of three types immune cells in the immune environment, and the results of KM analysis, we make the following inferences: The low expression of these 3 types of immune cells in the high-risk group may directly or indirectly reduce the responsiveness of the immune system, and thus the prognosis of sarcoma patients in the high-risk group is poorer than that of the low-risk group.

Although the role of hypoxia and immune-related prognostic signature in sarcoma patients has been initially investigated by statistical and bioinformatics analysis, the present study still has some shortcomings. First, other information such as tumor classification and treatment of sarcoma patients is not available from the TARGET database, which may impact on the prognosis of sarcoma patients. Secondly, the novel signature we built showed good performance. However, a large number of sarcoma samples are needed for external validation. Third, mechanisms related to hypoxia and immune correlation in sarcoma were explored in this study, but the underlying mechanisms affecting sarcoma remain unclear. Fourth, sarcomas are a highly heterogeneous class of tumors, and this study evaluated all sarcoma samples that meet the requirements from the TARGET database, so it was not possible to accurately assess the hypoxia and immune status of each sarcoma subtype.

\section{Conclusions}

In summary, we constructed and validated prognostic markers based on CMA1 and IGDCC3 to predict prognosis in sarcoma patients. In addition, our prognostic model provides a comprehensive view to elucidate the underlying mechanisms of sarcoma prognosis based on a combination of hypoxia and immune status. This prognostic profile helps clinical selection of individualized treatment strategies.

\section{Abbreviations}

PFS, progression-free survival; ICl, immune checkpoint inhibitor; PD-1, programmed cell death protein-1; PD-L1, programmed cell death protein-ligand 1; CTLA-4, cytotoxic T lymphocyte-associated antigen-4; 
ssGSEA, single sample Gene Set Enrichment Analysis; t-SNE, t-distributed Stochastic Neighbor Embedding; TARGET, Therapeutically Applicable Research To Generate Effective Treatments; HRDEGs, Hypoxia-related differentially expressed genes; HRGs, hypoxia-related genes; GSEA, Gene Set Enrichment Analysis; GO, gene ontology; HIF-1, hypoxia inducible factor 1; KEGG, Kyoto encyclopedia of genes and genomes; logFC, log fold change; IRDEGs, Immune-related differentially expressed genes; ESTIMATE, Estimation of Stromal and Immune cells in Malignant Tumor tissues using Expression; NES, normalized enrichment score; NOM p-val, nominal p-value; FDR q-val, false discovery rate q-value; TIMER, Tumor Immune Estimation Resource; TIM3, T-cell immunoglobulin mucin 3; MF, molecular function; BP, biological processes; $\mathrm{CC}$, cellular component; CMA1, Chymase 1; TME, tumor microenvironment.

\section{Declarations}

\section{ACKNOWLEDGMENTS}

We are very grateful for the contributions of TARGET database that provide information on cancer research, as well as all colleagues involved in the study.

\section{AUTHOR CONTRIBUTIONS}

Conceptualization, software, and methodology: Zhehong Li and Junqiang Wei. Data collection, modeling, validation, investigation: Zhehong Li, Junqiang Wei, Honghong Zheng, Xintian Gan, Mingze Song, Yafang Zhang, and Haiying Cao. Preparation of the first draft: Zhehong Li and Junqiang Wei. Supervision and funding acquisition: Yu Jin and Junqiang Wei. All authors have read and agreed to the published version of the manuscript.

\section{FUNDING}

We received no external funding for this study.

\section{DATA AVAILABILITY STATEMENT}

The authors confirm that the data supporting the findings of this study are available within the article.

\section{ETHICS APPROVAL AND CONSENT TO PARTICIPATE}

Not applicable.

\section{CONSENT FOR PUBLICATION}

Not applicable.

\section{COMPETING INTERESTS}

The authors declare that they have no competing interests. 


\section{References}

[1] Ng VY, Scharschmidt TJ, Mayerson JL, Fisher JL. Incidence and survival in sarcoma in the United States: a focus on musculoskeletal lesions. Anticancer Res. 2013 Jun;33(6):2597-604. PMID: 23749914.

[2] Hui JY. Epidemiology and Etiology of Sarcomas. Surg Clin North Am. 2016 Oct;96(5):901-14. doi: 10.1016/j.suc.2016.05.005. PMID: 27542634.

[3] Siegel RL, Miller KD, Jemal A. Cancer statistics, 2019. CA Cancer J Clin. 2019 Jan;69(1):7-34. doi: 10.3322/caac.21551. Epub 2019 Jan 8. PMID: 30620402.

[4] Miwa S, Yamamoto N, Hayashi K, Takeuchi A, Igarashi K, Tsuchiya H. Therapeutic Targets for Bone and Soft-Tissue Sarcomas. Int J Mol Sci. 2019 Jan 4;20(1):170. doi: 10.3390/ijms20010170. PMID: 30621224; PMCID: PMC6337155.

[5] Siegel RL, Miller KD, Fuchs HE, Jemal A. Cancer Statistics, 2021. CA Cancer J Clin. 2021 Jan;71(1):733. doi: 10.3322/caac.21654. Epub 2021 Jan 12. PMID: 33433946.

[6] Burns J, Wilding CP, L Jones R, H Huang P. Proteomic research in sarcomas - current status and future opportunities. Semin Cancer Biol. 2020 Apr;61:56-70. doi: 10.1016/j.semcancer.2019.11.003. Epub 2019 Nov 10. PMID: 31722230; PMCID: PMC7083238.

[7] Patel A, Sant S. Hypoxic tumor microenvironment: Opportunities to develop targeted therapies. Biotechnol Adv. 2016 Sep-Oct;34(5):803-812. doi: 10.1016/j.biotechadv.2016.04.005. Epub 2016 Apr 30. PMID: 27143654; PMCID: PMC4947437.

[8] Noman MZ, Hasmim M, Lequeux A, Xiao M, Duhem C, Chouaib S, Berchem G, Janji B. Improving Cancer Immunotherapy by Targeting the Hypoxic Tumor Microenvironment: New Opportunities and Challenges. Cells. 2019 Sep 14;8(9):1083. doi: 10.3390/cells8091083. PMID: 31540045; PMCID: PMC6770817.

[9] Eisinger-Mathason TS, Zhang M, Qiu Q, Skuli N, Nakazawa MS, Karakasheva T, Mucaj V, Shay JE, Stangenberg L, Sadri N, Puré E, Yoon SS, Kirsch DG, Simon MC. Hypoxia-dependent modification of collagen networks promotes sarcoma metastasis. Cancer Discov. 2013 Oct;3(10):1190-205. doi: 10.1158/2159-8290.CD-13-0118. Epub 2013 Aug 1. PMID: 23906982; PMCID: PMC3822914.

[10] Semenza GL. Cancer-stromal cell interactions mediated by hypoxia-inducible factors promote angiogenesis, lymphangiogenesis, and metastasis. Oncogene. 2013 Aug 29;32(35):4057-63. doi: 10.1038/onc.2012.578. Epub 2012 Dec 10. PMID: 23222717; PMCID: PMC4415159.

[11] Lee KH, Kim EY, Yun JS, Park YL, Do SI, Chae SW, Park CH. The prognostic and predictive value of tumor-infiltrating lymphocytes and hematologic parameters in patients with breast cancer. BMC Cancer. 2018 Oct 1;18(1):938. doi: 10.1186/s12885-018-4832-5. PMID: 30285668; PMCID: PMC6167816. 
[12] Groisberg R, Hong DS, Behrang A, Hess K, Janku F, Piha-Paul S, Naing A, Fu S, Benjamin R, Patel S, Somaiah N, Conley A, Meric-Bernstam F, Subbiah V. Characteristics and outcomes of patients with advanced sarcoma enrolled in early phase immunotherapy trials. J Immunother Cancer. 2017 Dec 19;5(1):100. doi: 10.1186/s40425-017-0301-y. PMID: 29254498; PMCID: PMC5735899.

[13] Gubin MM, Zhang X, Schuster H, Caron E, Ward JP, Noguchi T, Ivanova Y, Hundal J, Arthur CD, Krebber WJ, Mulder GE, Toebes M, Vesely MD, Lam SS, Korman AJ, Allison JP, Freeman GJ, Sharpe AH, Pearce EL, Schumacher TN, Aebersold R, Rammensee HG, Melief CJ, Mardis ER, Gillanders WE, Artyomov MN, Schreiber RD. Checkpoint blockade cancer immunotherapy targets tumour-specific mutant antigens. Nature. 2014 Nov 27;515(7528):577-81. doi: 10.1038/nature13988. PMID: 25428507; PMCID: PMC4279952.

[14] Galon J, Bruni D. Approaches to treat immune hot, altered and cold tumours with combination immunotherapies. Nat Rev Drug Discov. 2019 Mar;18(3):197-218. doi: 10.1038/s41573-018-0007-y. PMID: 30610226.

[15] Sharma P, Allison JP. The future of immune checkpoint therapy. Science. 2015 Apr 3;348(6230):5661. doi: 10.1126/science.aaa8172. PMID: 25838373.

[16] Riera-Domingo C, Audigé A, Granja S, Cheng WC, Ho PC, Baltazar F, Stockmann C, Mazzone M. Immunity, Hypoxia, and Metabolism-the Ménage à Trois of Cancer: Implications for Immunotherapy. Physiol Rev. 2020 Jan 1;100(1):1-102. doi: 10.1152/physrev.00018.2019. Epub 2019 Aug 15. PMID: 31414610.

[17] StahI D, Gentles AJ, Thiele R, Gütgemann I. Prognostic profiling of the immune cell microenvironment in Ewing's Sarcoma Family of Tumors. Oncoimmunology. 2019 Oct 13;8(12):e1674113. doi:

10.1080/2162402X.2019.1674113. PMID: 31741777; PMCID: PMC6844324.

[18] Ma L, Shan W, Ding X, Yang P, Rozjan A, Yao Q. Intermittent hypoxia induces tumor immune escape in murine S180 solid tumors via the upregulation of TGF- $\beta 1$ in mice. Sleep Breath. 2021 Jun;25(2):719-726. doi: 10.1007/s11325-020-02166-2. Epub 2020 Aug 25. PMID: 32840731.

[19] Prudowsky ZD, Yustein JT. Recent Insights into Therapy Resistance in Osteosarcoma. Cancers (Basel). 2020 Dec 30;13(1):83. doi: 10.3390/cancers13010083. PMID: 33396725; PMCID: PMC7795058.

[20] Hu B, Yang XB, Sang XT. Development and Verification of the Hypoxia-Related and ImmuneAssociated Prognosis Signature for Hepatocellular Carcinoma. J Hepatocell Carcinoma. 2020 Nov 11;7:315-330. doi: 10.2147/JHC.S272109. PMID: 33204664; PMCID: PMC7667586.

[21] Yoshikawa Y, Yamanouchi K. Effect of papaverine treatment on replication of measles virus in human neural and nonneural cells. J Virol. 1984 May;50(2):489-96. doi: 10.1128/JVI.50.2.489-496.1984. PMID: 6708172; PMCID: PMC255654. 
[22] Yang Z, Yang X, Xu S, Jin P, Li X, Wei X, Liu D, Huang K, Long S, Wang Y, Sun C, Chen G, Hu J, Meng L, Ma D, Gao Q. Reprogramming of stromal fibroblasts by SNAI2 contributes to tumor desmoplasia and ovarian cancer progression. Mol Cancer. 2017 Oct 17;16(1):163. doi: 10.1186/s12943-017-0732-6. PMID: 29041931; PMCID: PMC5645935.

[23] Xiao B, Liu L, Li A, Xiang C, Wang P, Li H, Xiao T. Identification and Verification of Immune-Related Gene Prognostic Signature Based on ssGSEA for Osteosarcoma. Front Oncol. 2020 Dec 15;10:607622. doi: 10.3389/fonc.2020.607622. PMID: 33384961; PMCID: PMC7771722.

[24] Yoshihara K, Shahmoradgoli M, Martínez E, Vegesna R, Kim H, Torres-Garcia W, Treviño V, Shen H, Laird PW, Levine DA, Carter SL, Getz G, Stemke-Hale K, Mills GB, Verhaak RG. Inferring tumour purity and stromal and immune cell admixture from expression data. Nat Commun. 2013;4:2612. doi:

10.1038/ncomms3612. PMID: 24113773; PMCID: PMC3826632.

[25] Li T, Fan J, Wang B, Traugh N, Chen Q, Liu JS, Li B, Liu XS. TIMER: A Web Server for Comprehensive Analysis of Tumor-Infiltrating Immune Cells. Cancer Res. 2017 Nov 1;77(21):e108-e110. doi:

10.1158/0008-5472.CAN-17-0307. PMID: 29092952; PMCID: PMC6042652.

[26] Gentles AJ, Newman AM, Liu CL, Bratman SV, Feng W, Kim D, Nair VS, Xu Y, Khuong A, Hoang CD, Diehn M, West RB, Plevritis SK, Alizadeh AA. The prognostic landscape of genes and infiltrating immune cells across human cancers. Nat Med. 2015 Aug;21(8):938-945. doi: 10.1038/nm.3909. Epub $2015 \mathrm{Jul}$ 20. PMID: 26193342; PMCID: PMC4852857.

[27] Semenza GL. Targeting HIF-1 for cancer therapy. Nat Rev Cancer. 2003 Oct;3(10):721-32. doi: 10.1038/nrc1187. PMID: 13130303.

[28] Ehnman M, Chaabane W, Haglund F, Tsagkozis P. The Tumor Microenvironment of Pediatric Sarcoma: Mesenchymal Mechanisms Regulating Cell Migration and Metastasis. Curr Oncol Rep. 2019 Aug 15;21(10):90. doi: 10.1007/s11912-019-0839-6. PMID: 31418125; PMCID: PMC6695368.

[29] Yang L, Forker L, Irlam JJ, Pillay N, Choudhury A, West CML. Correction: Validation of a hypoxia related gene signature in multiple soft tissue sarcoma cohorts. Oncotarget. 2019 Mar 8;10(20):2007. doi: 10.18632/oncotarget.26783. Erratum for: Oncotarget. 2017 Dec 12;9(3):3946-3955. PMID: 30956781; PMCID: PMC6443018.

[30] Detwiller KY, Fernando NT, Segal NH, Ryeom SW, D'Amore PA, Yoon SS. Analysis of hypoxia-related gene expression in sarcomas and effect of hypoxia on RNA interference of vascular endothelial cell growth factor A. Cancer Res. 2005 Jul 1;65(13):5881-9. doi: 10.1158/0008-5472.CAN-04-4078. PMID: 15994966.

[31] Marin JJG, Macias RIR, Monte MJ, Herraez E, Peleteiro-Vigil A, Blas BS, Sanchon-Sanchez P, Temprano AG, Espinosa-Escudero RA, Lozano E, Briz O, Romero MR. Cellular Mechanisms Accounting for 
the Refractoriness of Colorectal Carcinoma to Pharmacological Treatment. Cancers (Basel). 2020 Sep 11;12(9):2605. doi: 10.3390/cancers12092605. PMID: 32933095; PMCID: PMC7563523.

[32] Xiao W, Sun G, Fan T, Liu J, Zhang N, Zhao L, Zhong R. Reductive Activity and Mechanism of Hypoxia- Targeted AGT Inhibitors: An Experimental and Theoretical Investigation. Int J Mol Sci. 2019 Dec 13;20(24):6308. doi: 10.3390/ijms20246308. PMID: 31847200; PMCID: PMC6941096.

[33] Shao C, Yang F, Miao S, Liu W, Wang C, Shu Y, Shen H. Role of hypoxia-induced exosomes in tumor biology. Mol Cancer. 2018 Aug 11;17(1):120. doi: 10.1186/s12943-018-0869-y. PMID: 30098600; PMCID: PMC6087002.

[34] Stahl D, Gentles AJ, Thiele R, Gütgemann I. Prognostic profiling of the immune cell microenvironment in Ewing's Sarcoma Family of Tumors. Oncoimmunology. 2019 Oct 13;8(12):e1674113. doi: 10.1080/2162402X.2019.1674113. PMID: 31741777; PMCID: PMC6844324.

[35] Hu C, Chen B, Huang Z, Liu C, Ye L, Wang C, Tong Y, Yang J, Zhao C. Comprehensive profiling of immune-related genes in soft tissue sarcoma patients. J Transl Med. 2020 Sep 1;18(1):337. doi: 10.1186/s12967-020-02512-8. PMID: 32873319; PMCID: PMC7465445.

[36] Kobak D, Berens P. The art of using t-SNE for single-cell transcriptomics. Nat Commun. 2019 Nov 28;10(1):5416. doi: 10.1038/s41467-019-13056-x. PMID: 31780648; PMCID: PMC6882829.

[37] Fu Y, Bao Q, Liu Z, He G, Wen J, Liu Q, Xu Y, Jin Z, Zhang W. Development and Validation of a Hypoxia-Associated Prognostic Signature Related to Osteosarcoma Metastasis and Immune Infiltration. Front Cell Dev Biol. 2021 Mar 18;9:633607. doi: 10.3389/fcell.2021.633607. PMID: 33816483; PMCID: PMC8012854.

[38] Hou C, Cai H, Zhu Y, Huang S, Song F, Hou J. Development and Validation of Autophagy-Related Gene Signature and Nomogram for Predicting Survival in Oral Squamous Cell Carcinoma. Front Oncol. 2020 Oct 16;10:558596. doi: 10.3389/fonc.2020.558596. PMID: 33178587; PMCID: PMC7596585.

[39] Yoshihara K, Shahmoradgoli M, Martínez E, Vegesna R, Kim H, Torres-Garcia W, Treviño V, Shen H, Laird PW, Levine DA, Carter SL, Getz G, Stemke-Hale K, Mills GB, Verhaak RG. Inferring tumour purity and stromal and immune cell admixture from expression data. Nat Commun. 2013;4:2612. doi:

10.1038/ncomms3612. PMID: 24113773; PMCID: PMC3826632.

[40] Sharma S, Rajan UM, Kumar A, Soni A, Ghosh B. A novel (TG)n(GA)m repeat polymorphism 254 bp downstream of the mast cell chymase (CMA1) gene is associated with atopic asthma and total serum IgE levels. J Hum Genet. 2005;50(6):276-282. doi: 10.1007/s10038-005-0252-x. Epub 2005 May 28. PMID: 15924217.

[41] Ono K, Kokubo Y, Mannami T, Inamoto N, Shioji K, Iwai N. Heterozygous disruption of CMA1 does not affect blood pressure. J Hypertens. 2004 Jan;22(1):103-9. doi: 10.1097/00004872-200401000-00019. 
PMID: 15106801.

[42] Shi S, Ye S, Mao J, Ru Y, Lu Y, Wu X, Xu M, Zhu T, Wang Y, Chen Y, Tang X, Xi Y. CMA1 is potent prognostic marker and associates with immune infiltration in gastric cancer. Autoimmunity. 2020 Jun;53(4):210-217. doi: 10.1080/08916934.2020.1735371. Epub 2020 Mar 4. PMID: 32129682.

[43] Chong A, Teo JX, Ban KH. Distinct epigenetic signatures elucidate enhancer-gene relationships that delineate CIMP and non-CIMP colorectal cancers. Oncotarget. 2016 May 10;7(19):28027-39. doi: 10.18632/oncotarget.8473. PMID: 27049830; PMCID: PMC5053707.

[44] D'Angelo SP, Mahoney MR, Van Tine BA, Atkins J, Milhem MM, Jahagirdar BN, Antonescu CR, Horvath E, Tap WD, Schwartz GK, Streicher H. Nivolumab with or without ipilimumab treatment for metastatic sarcoma (Alliance A091401): two open-label, non-comparative, randomised, phase 2 trials. Lancet Oncol. 2018 Mar;19(3):416-426. doi: 10.1016/S1470-2045(18)30006-8. Epub 2018 Jan 19. PMID: 29370992; PMCID: PMC6126546.

[45] Chang CH, Qiu J, O'Sullivan D, Buck MD, Noguchi T, Curtis JD, Chen Q, Gindin M, Gubin MM, van der Windt GJ, Tonc E, Schreiber RD, Pearce EJ, Pearce EL. Metabolic Competition in the Tumor Microenvironment Is a Driver of Cancer Progression. Cell. 2015 Sep 10;162(6):1229-41. doi: 10.1016/j.cell.2015.08.016. Epub 2015 Aug 27. PMID: 26321679; PMCID: PMC4864363.

[46] Ngiow SF, von Scheidt B, Akiba H, Yagita H, Teng MW, Smyth MJ. Anti-TIM3 antibody promotes T cell IFN-y-mediated antitumor immunity and suppresses established tumors. Cancer Res. 2011 May 15;71(10):3540-51. doi: 10.1158/0008-5472.CAN-11-0096. Epub 2011 Mar 23. PMID: 21430066.

[47] Petitprez F, de Reyniès A, Keung EZ, Chen TW, Sun CM, Calderaro J, Jeng YM, Hsiao LP, Lacroix L, Bougoüin A, Moreira M, Lacroix G, Natario I, Adam J, Lucchesi C, Laizet YH, Toulmonde M, Burgess MA, Bolejack V, Reinke D, Wani KM, Wang WL, Lazar AJ, Roland CL, Wargo JA, Italiano A, Sautès-Fridman C, Tawbi HA, Fridman WH. B cells are associated with survival and immunotherapy response in sarcoma. Nature. 2020 Jan;577(7791):556-560. doi: 10.1038/s41586-019-1906-8. Epub 2020 Jan 15. PMID: 31942077.

[48] Topalian SL, Hodi FS, Brahmer JR, Gettinger SN, Smith DC, McDermott DF, Powderly JD, Carvajal RD, Sosman JA, Atkins MB, Leming PD, Spigel DR, Antonia SJ, Horn L, Drake CG, Pardoll DM, Chen L, Sharfman WH, Anders RA, Taube JM, McMiller TL, Xu H, Korman AJ, Jure-Kunkel M, Agrawal S, McDonald D, Kollia GD, Gupta A, Wigginton JM, Sznol M. Safety, activity, and immune correlates of antiPD-1 antibody in cancer. N Engl J Med. 2012 Jun 28;366(26):2443-54. doi: 10.1056/NEJMoa1200690. Epub 2012 Jun 2. PMID: 22658127; PMCID: PMC3544539.

[49] Klaver Y, Rijnders M, Oostvogels A, Wijers R, Smid M, Grünhagen D, Verhoef C, Sleijfer S, Lamers C, Debets R. Differential quantities of immune checkpoint-expressing CD8 T cells in soft tissue sarcoma subtypes. J Immunother Cancer. 2020 Aug;8(2):e000271. doi: 10.1136/jitc-2019-000271. Erratum in: J Immunother Cancer. 2020 Sep;8(2): PMID: 32792357; PMCID: PMC7430493. 
Figures

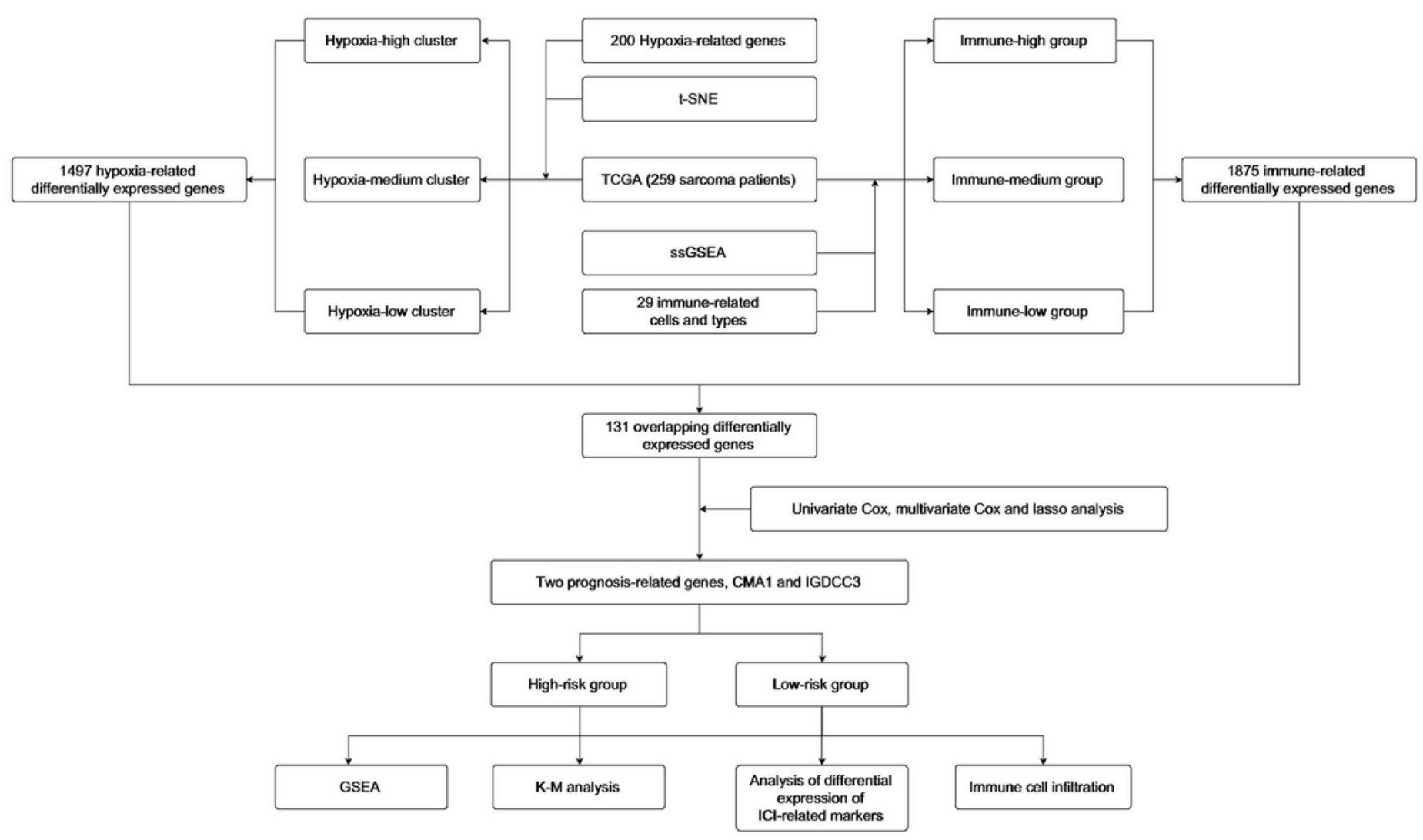

Figure 1

The design idea and workflow. 

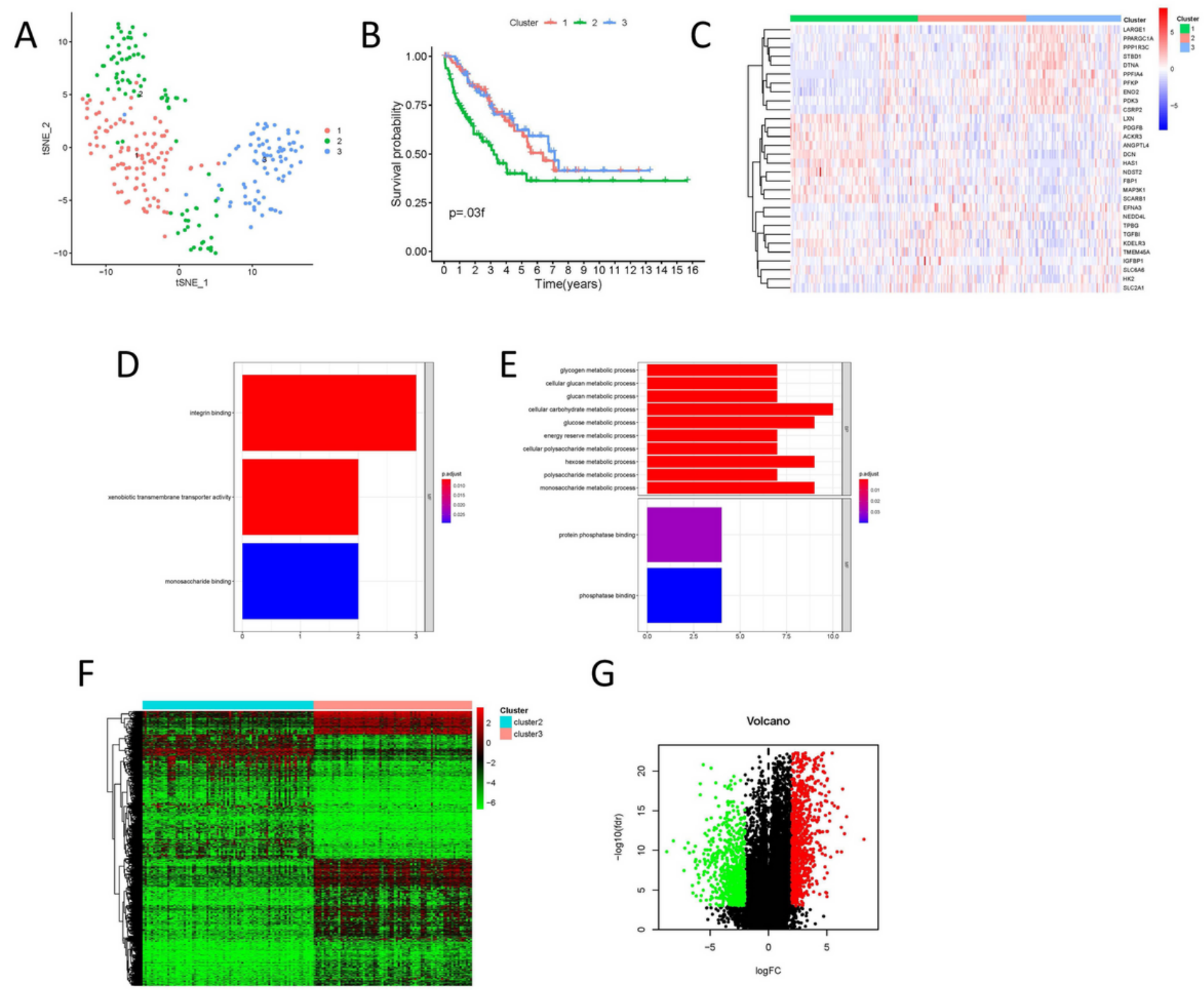

\section{Figure 2}

Identification of hypoxia status and hypoxia-related differentially expressed genes (HRDEGs). (A) Scatter plot for three different clusters by the nonlinear dimensionality reduction algorithm t-SNE based on hypoxia-related genes set. (B) Kaplan-Meier survival curve of overall survival for sarcoma patients in three clusters. (C) Heat map of the top 10 marker genes for three cluster. The Gene Ontology (GO) analysis of the marker genes in Cluster 2 (D) and Cluster $3(E)$. Heat map (F) and volcano map (G) show the HRDEGs in high and low hypoxia groups. HRDEGs, hypoxia-related differentially expressed genes; GO, Gene Ontology. 
A

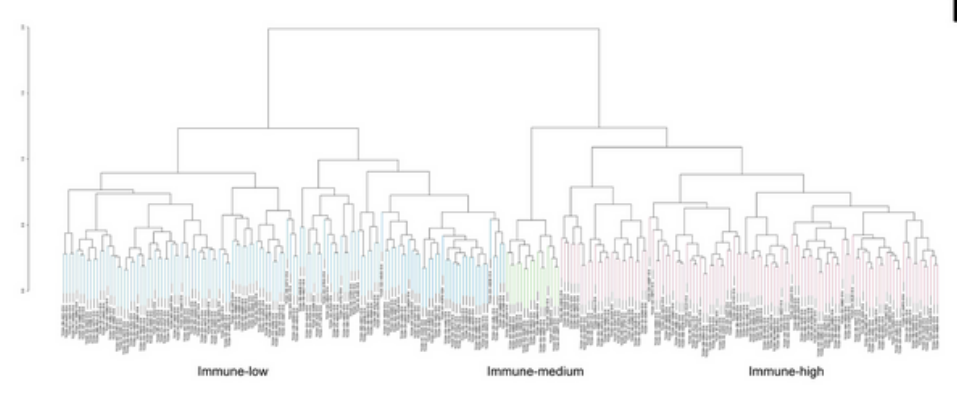

C

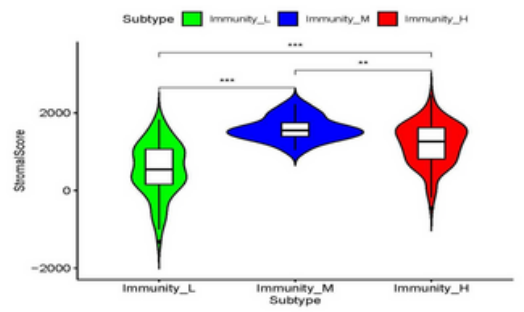

$\mathrm{F}$

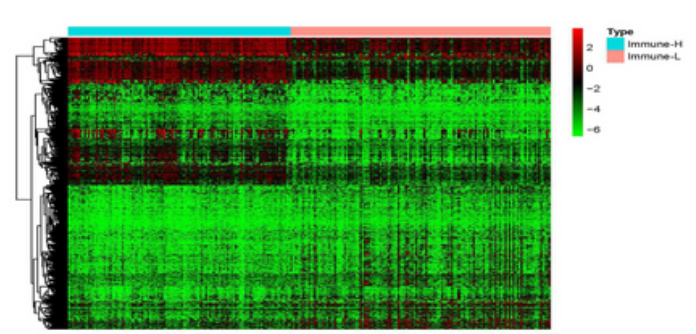

G
B

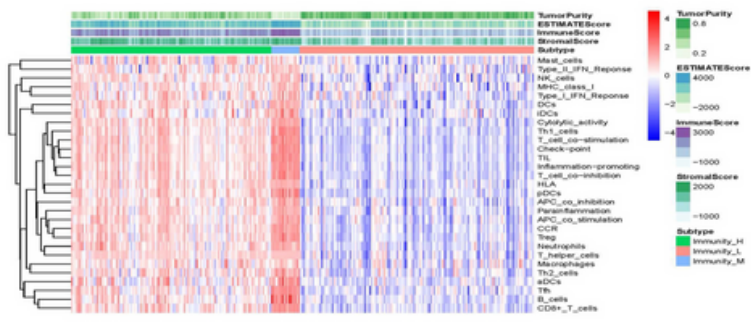

$\mathrm{E}$

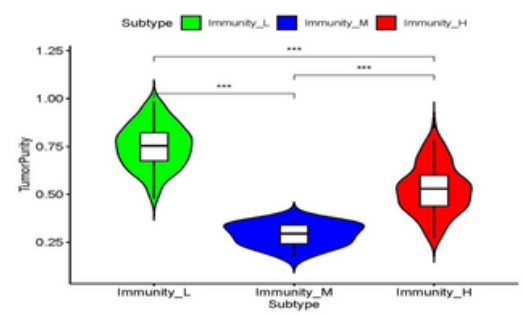

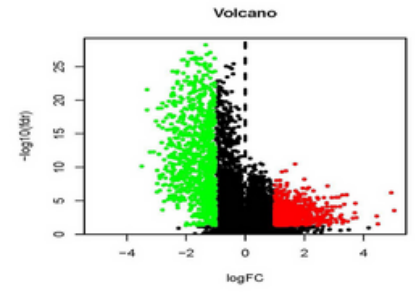

Figure 3

Identification of immune status and immune-related differentially expressed genes (IRDEGs). (A) Cluster map for the hierarchical clustering of sarcoma by the "GSVA" package based on 29 immune-related cells and types. (B) Different immune status of the three clusters. Comparison of (C) stromal score, (D) immune score and (E) tumor purity between high- and low-immune clusters are shown. Heat map (F) and volcano map (G) show the IRDEGs in high and low-immune groups. IRDEGs, immune-related differentially expressed genes. 
A

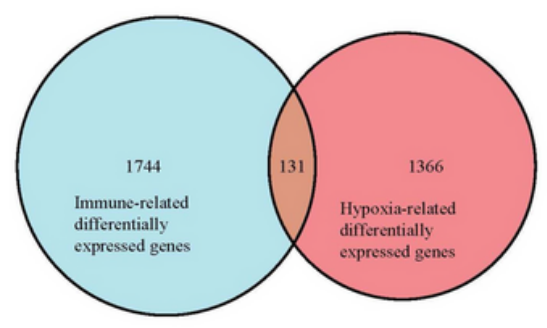

D

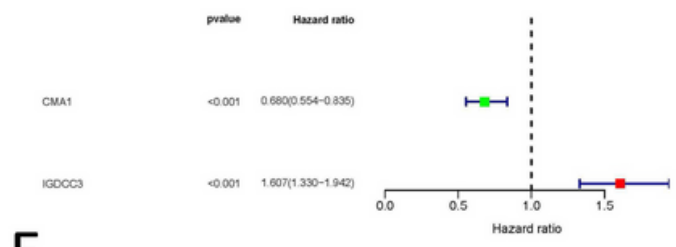

E

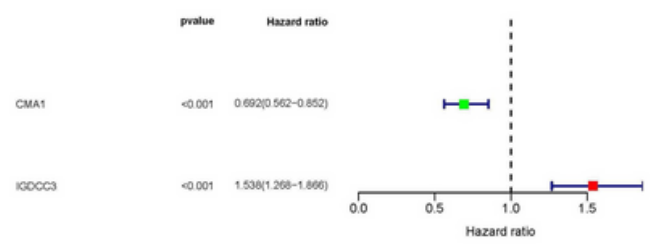

B

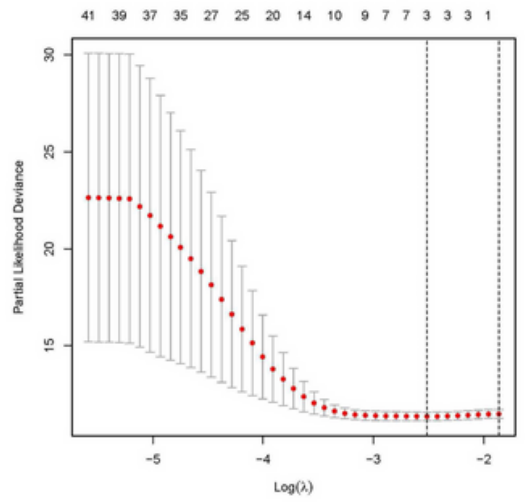

$\mathrm{F}$

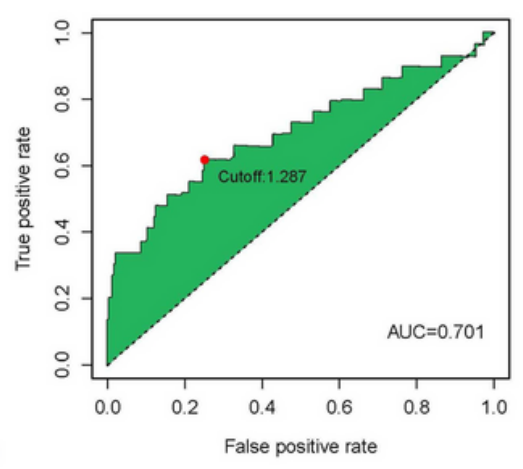

C

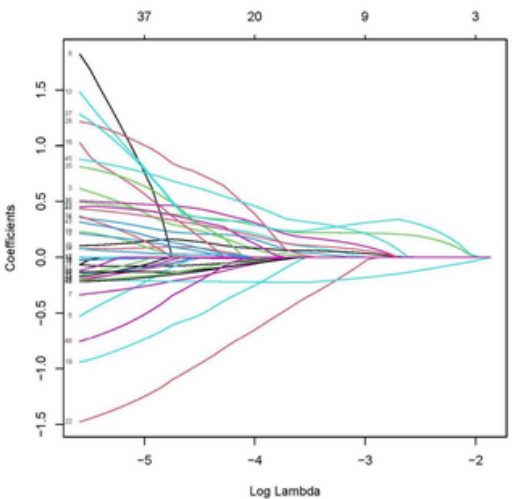

G

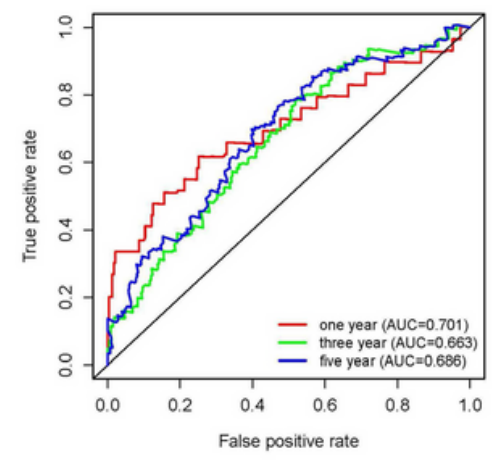

$\mathrm{H}$

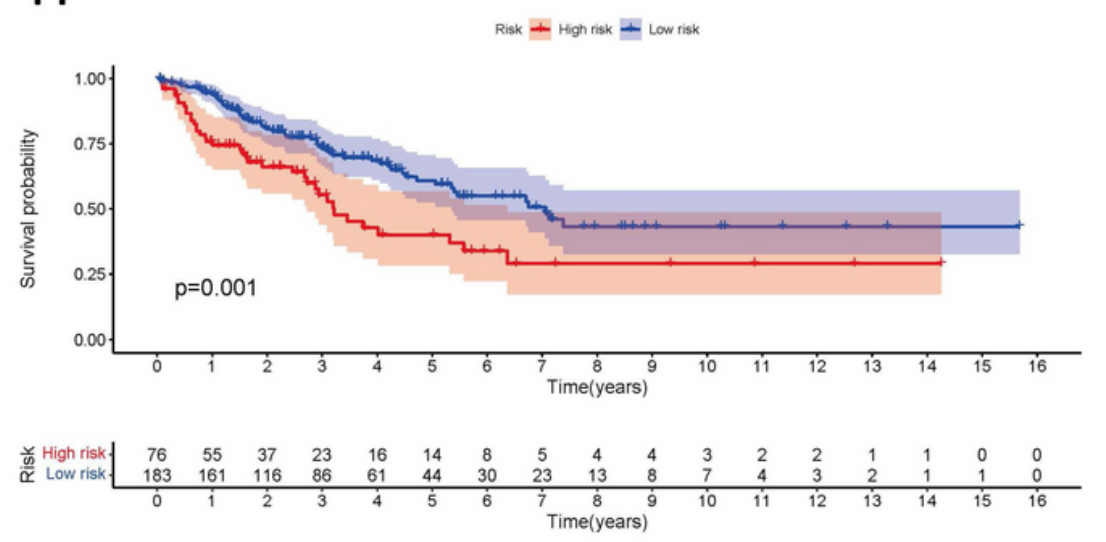

\section{Figure 4}

Development of risk scoring signatures and evaluation of predictive models. (A) Venn plot for overlapping genes obtained by taking intersections of HRDEGs and IRDEGs. (B-C) The results of LASSO analysis. The results of $(D)$ univariate and $(E)$ multivariate Cox regression analysis. $(F)$ the prognostic signature's ROC curves with an optimal cut-off point of 1.287 (red point). (G) The 1-, 3-, and 5-years ROC curves of prognostic signature. $(\mathrm{H})$ the K-M analysis between high and low-risk group. HRDEGs, hypoxia-related differentially expressed genes; IRDEGs, immune-related differentially expressed genes; LASSO, Least 
absolute shrinkage and selection operator; ROC, receiver operating characteristic curve; K-M, KaplanMeier.

A

$\begin{array}{lrr} & \text { pvalue } & \text { Hazard ratio } \\ \text { Age } & 0.008 & 1.021(1.005-1.038) \\ \text { Gender } & 0.477 & 0.865(0.579-1.291) \\ \text { Race } & 0.021 & 1.950(1.104-3.447) \\ \text { Site } & 0.262 & 1.261(0.841-1.891) \\ \text { Margin } & 0.184 & 1.234(0.905-1.683) \\ \text { Metastasis } & 0.677 & 1.058(0.812-1.377) \\ \text { Riskscore } & <0.001 & 1.176(1.103-1.254)\end{array}$

C

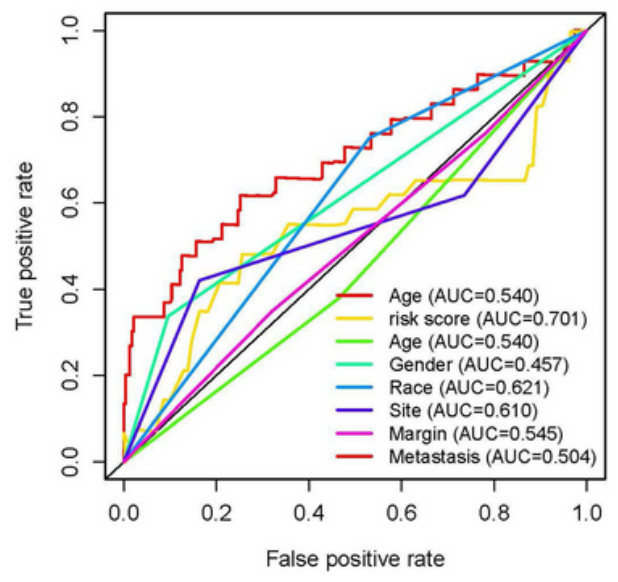

B
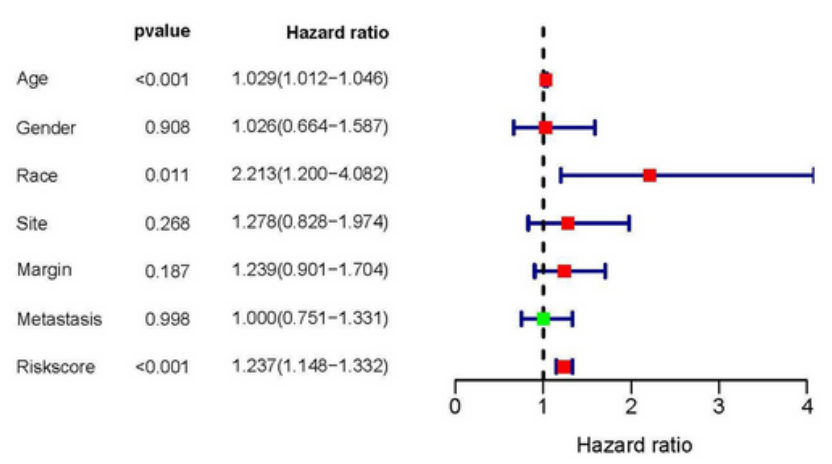

$\mathrm{D}$

\section{Figure 5}

Relationship between prognostic signature and clinicopathological features. Univariate $(A)$ and multivariate (B) Cox regression analysis between clinicopathological characteristics and prognostic signature. (C) The ROC curve for clinicopathological characteristics and prognostic signature. (D) The distributions of gene expression, risk score, and survival status in high and low-risk group. ROC, receiver operating characteristic curve. 
A
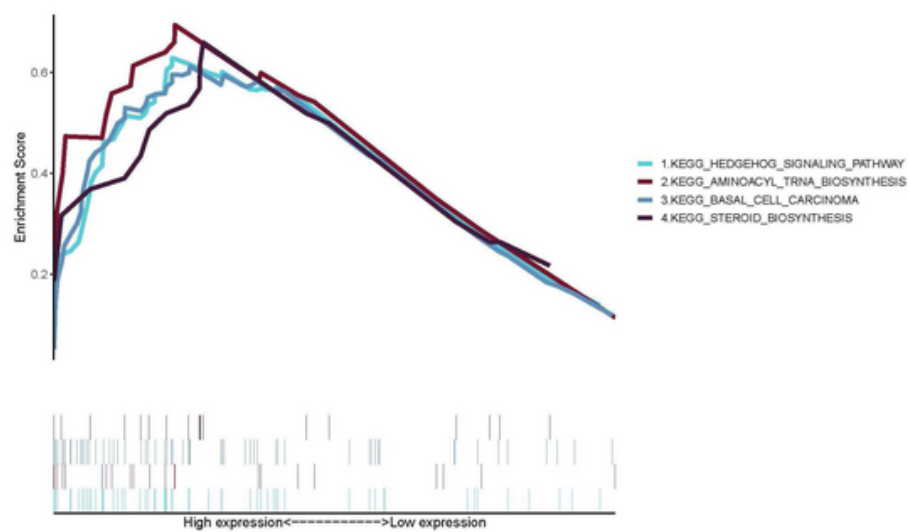

C
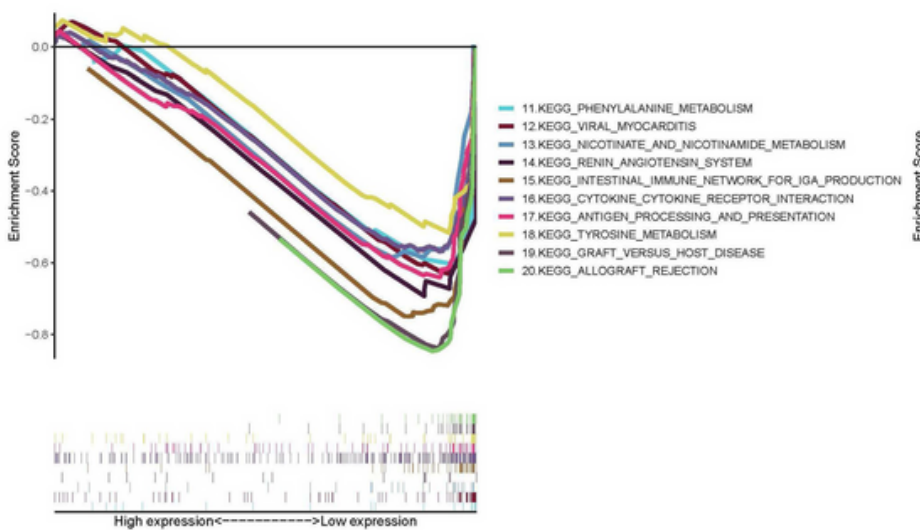

E
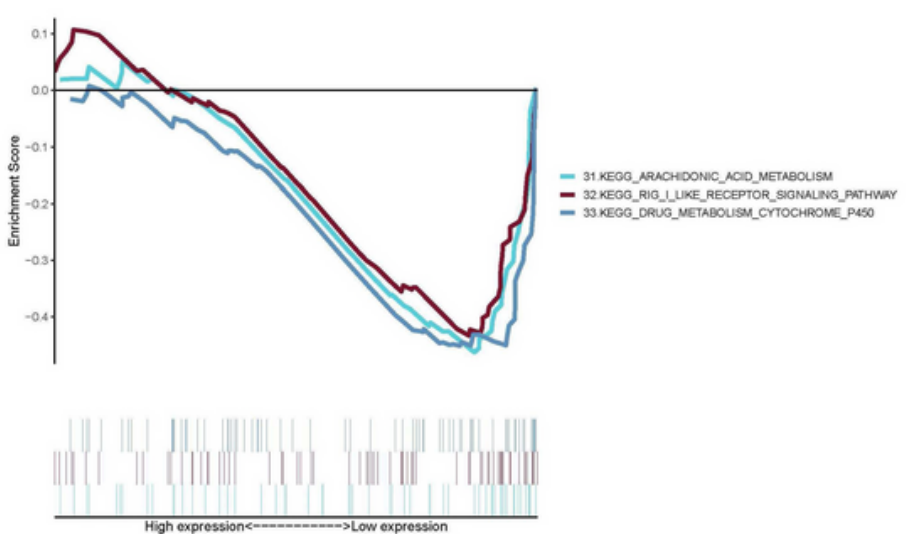

B
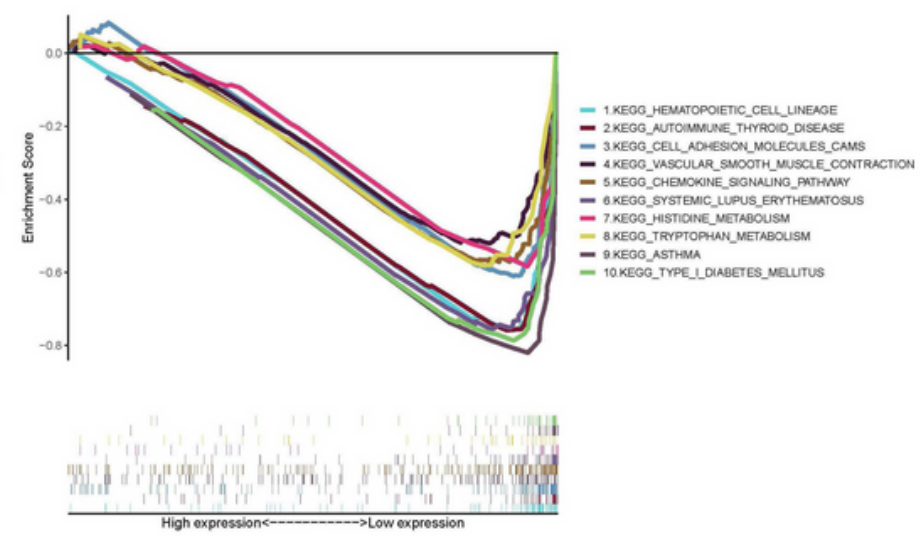

D

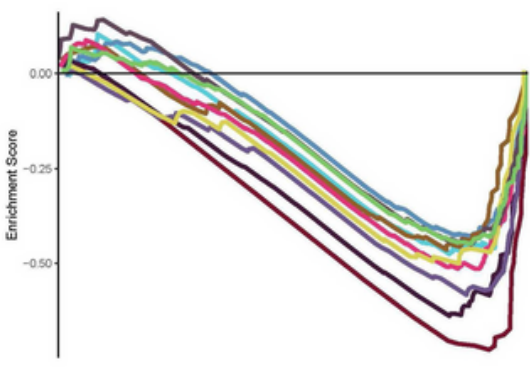

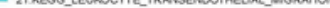

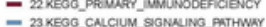

- 24 KEgG LESTMANA JNEECTION

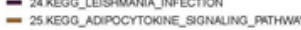
- 20 KEOG_COMPLEMENT ANO_CONOULATION CASCADES - 27 KEGG_TCELL_RECEPTOR_SGNAUNG_ANTHMAT

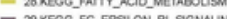

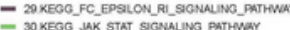

\section{Figure 6}

Gene set enrichment analysis (GSEA). (A) Enrichment of four signaling pathways based on the high-risk group. (B-E) Enrichment of Thirty-three signaling pathways based on the low-risk group. GSEA, Gene set enrichment analysis. 
A

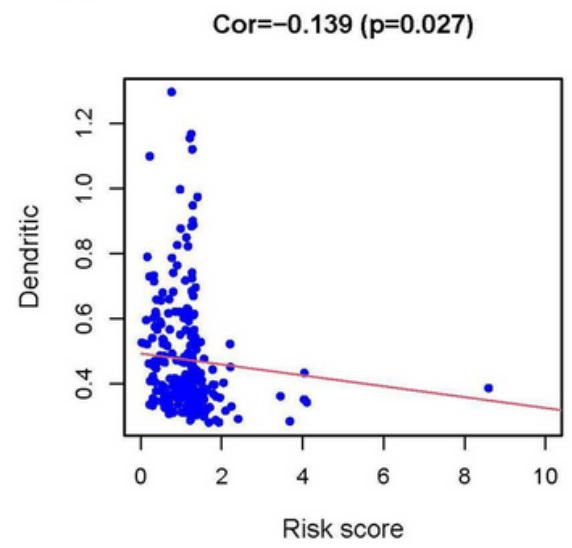

D

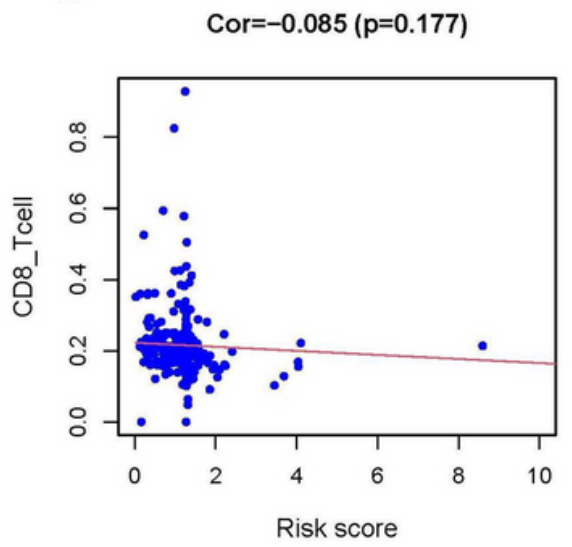

B

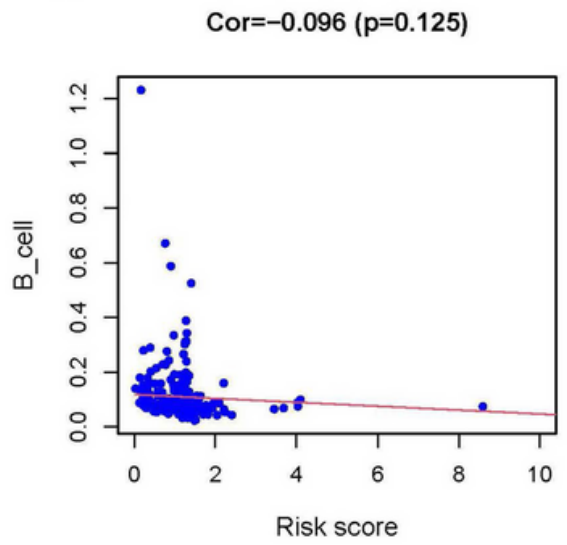

$E$

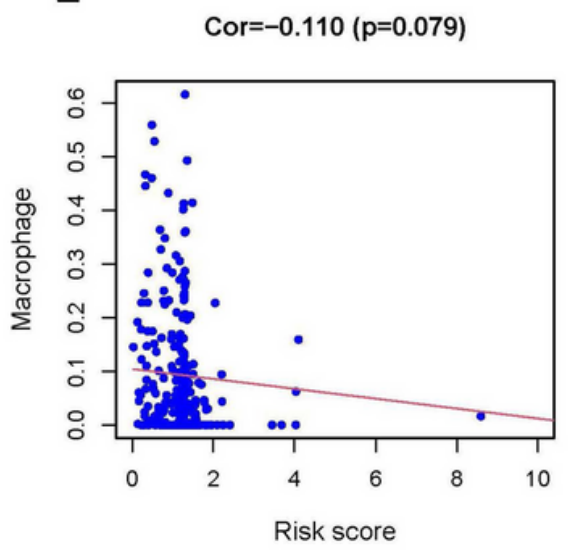

C

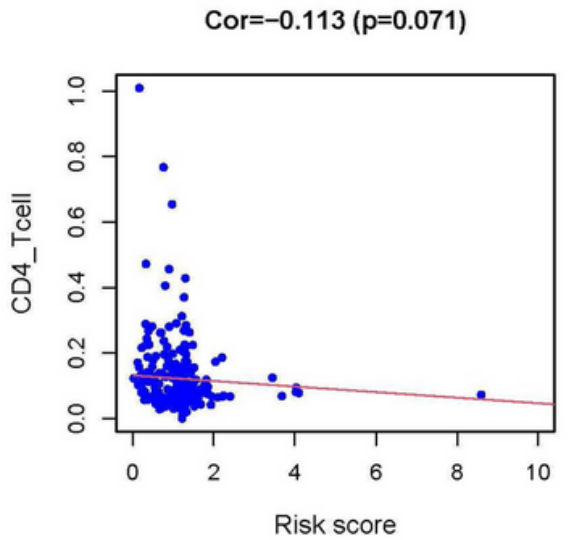

$\mathrm{F}$

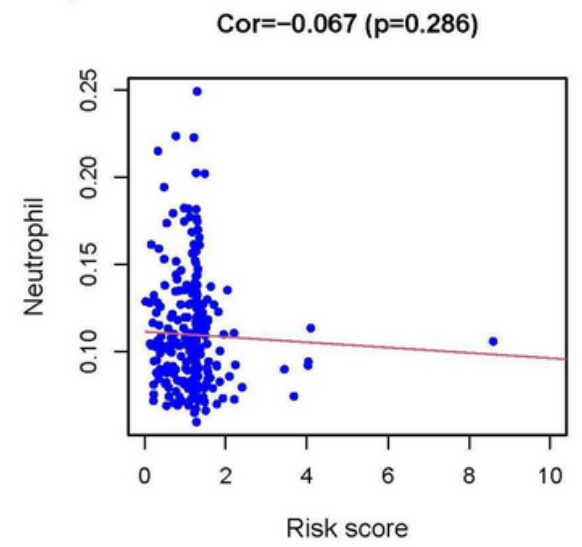

Figure 7

Relationships between the prognostic signature and infiltration abundances of six types of immune cell. The correlation was performed by using Pearson correlatioh analysis. (A) Dendritic Cells, (B) B cells, (C) CD4+ T cells, (D) CD8+ T cells, (E) Neutrophils, and (F) Macrophages. 

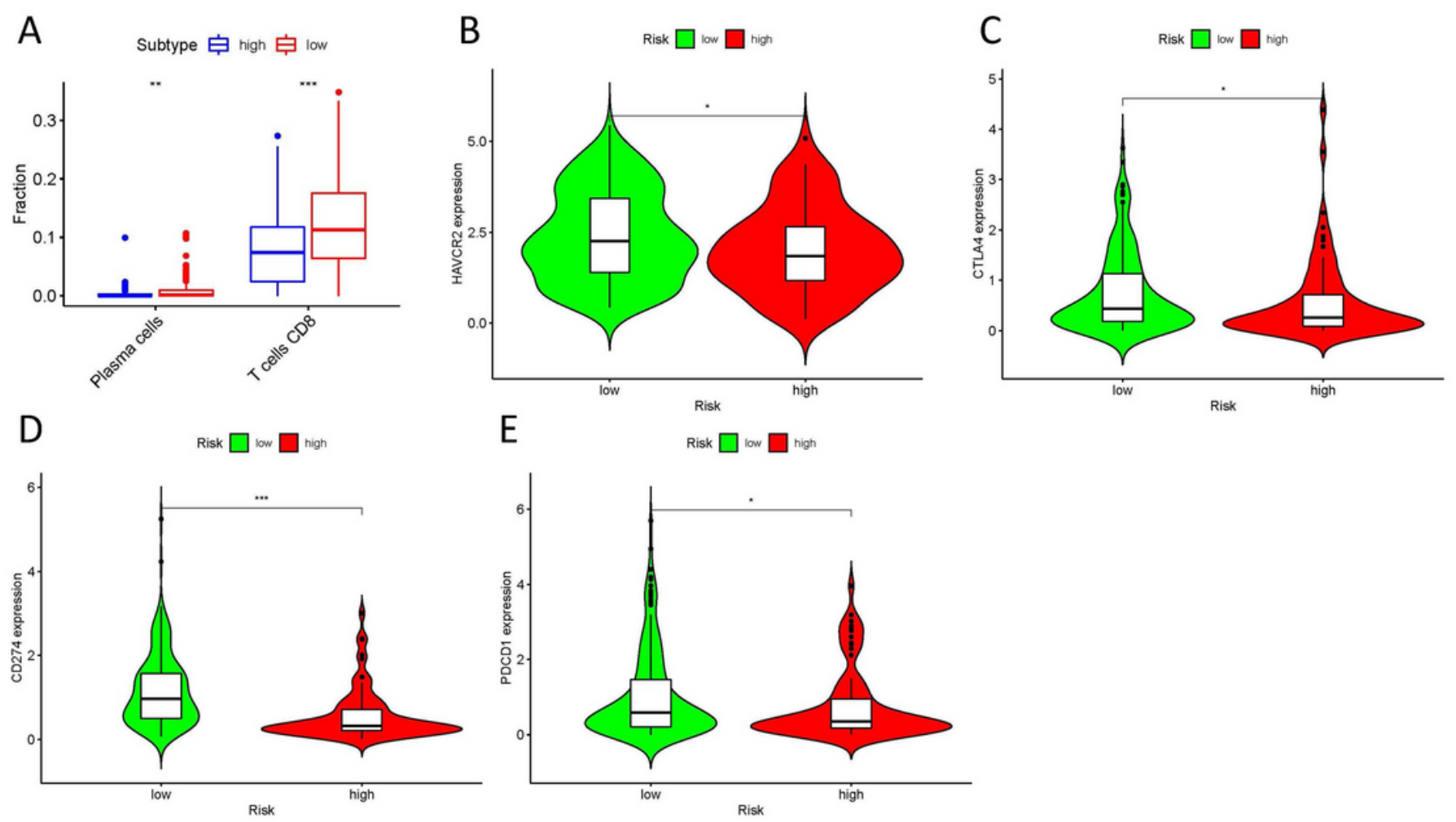

\section{Figure 8}

Analysis of different immune status in high- and low-risk groups. (A) Box plots visualizing significantly different immune cells between high-risk and low-risk patients. Box plot shows the difference in (B) HAVCR2, (C) CTLA4, (D) CD274, (E) PDCD1 between high-risk and low-risk group.

\section{Supplementary Files}

This is a list of supplementary files associated with this preprint. Click to download.

- SupplementaryFigure1.jpg

- SupplementaryFigure2.jpg

- SupplementaryFigure3.jpg

- SupplementaryFigure4.jpg

- SupplementaryFigure5.jpg

- SupplementaryFigure6.jpg

- SupplementaryFigure7.jpg

- SupplementaryTable1.xlsx

- SupplementaryTable2.xls

- SupplementaryTable3.xlsx 
- SupplementaryTable4.xIsx

- SupplementaryTable5.xlsx

- SupplementaryTable6.xIsx

- SupplementaryTable7.xlsx

- SupplementaryTable8.xIsx

- SupplementaryTable9.xIsx

- SupplementaryTable10.xlsx

- SupplementaryTable11.xlsx 